\title{
A Sound and Complete Axiomatisation for Spatio-Temporal Specification Language
}

\author{
Tengfei Li, Jing Liu*, Dongdong An, Haiying Sun \\ Shanghai Key Laboratory of Trustworthy Computing, East China Normal University, Shanghai, China
}

\begin{abstract}
Specifying spatio-temporal aspects is one of the important areas in cyber-physical systems. Spatio-temporal logic with changes of truth value in discrete time and dense time has been researched, but a combination of spatial and temporal components with changes of spatial entities in dense time hasn't been well-done. The major problem is dense time and real-valued variables of the spatio-temporal properties of cyber-physical systems. In this paper, we propose a spatio-temporal specification language, named STSL, which integrates Signal Temporal Logic (STL) with a spatial logic $\mathcal{S} 4_{u}$ to deal with the changes of realvalues spatial entities in dense time. The combined language is divided into two formalisms, $S T S L_{P C}$ and $S T S L_{O C}$, which is applied to interpret the Boolean semantics and quantitative semantics, respectively. The syntax of the two formalism and the corresponding semantics are provided. Besides, we present a Hilbert-style axiomatization for the proposed STSL and provide the soundness and completeness result by the spatio-temporal extension of maximal consistent set and canonical model.

Index Terms-Signal Temporal Logic (STL) $\mathcal{S} 4_{u}$ SpatioTemporal Specification Language (STSL) $S T S L_{P C}$ $S T S L_{O C}$ Soundness Completeness Axiomatization System
\end{abstract}

\section{INTRODUCTION}

It is a challenging work to model cyber-physical systems, not only because cyber-physical systems integrate cyber systems, physical environment and the interactive part of them, but also because cyber-physical systems combine temporal and spatial aspects, discrete and continuous behavior, and uncertainty. Describing spatio-temporal aspects is one of the important areas in cyber-physical systems. Many works has been done with hybrid [1] and stochastic behaviors of cyberphysical systems, but fewer researchers concentrate on spatiotemporal aspects. The major problem is multidimensional expressiveness and expensive verifiability for modeling and analysis of the spatio-temporal behaviors of cyber-physical systems.

This work aims at building a spatio-temporal specification language (STSL) by solving spatio-temporal constraints concerning dense time and real-valued variables, as an intelligent object in physical environment is provided with changes in specified space and continuous time. More specifically, we confine ourselves to the combination of topological space and time constraints with real-valued interval, which may

*Corresponding Author: jliu@ @ei.ecnu.edu.cn

DOI reference number: 10.18293/SEKE2019-222 be an open, half-open and half-closed, half-closed and halfopen, or closed interval in a flow of time. We adopt the modal spatial logic $\mathcal{S} 4_{u}$ to express topological constraints, which is one of the most influential formalism and the most expressiveness for topological relations. As for signal temporal logic (STL) [2], [3], there are two approaches that can cope with signals, quantitative semantics and Boolean semantics. Quantitative semantics obtains real-valued signals from satisfaction degree of a trace in real-valued interval. While Boolean semantics evaluates Boolean signals from a trace which can be booleanized through a set of threshold predicates.

Combining spatio-temporal constraints from temporal logics and modal spatial logics is a very important problem. Given a spatio-temporal model $\mathfrak{M}$ and a STSL formula $\varphi$, the satisfiability problem of the formula $\varphi$ is to $\operatorname{check}$ if $\varphi$ is satisfiable in model $\mathfrak{M}$.

Since the changes of spatial entities and the flows of time are not independent, the combination between modal spatial logics and temporal logics is divided into two formalism, $S T S L_{P C}$ and $S T S L_{O C}$. $S T S L_{P C}$ means the changes of spatial propositions over time, while $S T S L_{O C}$ represents the changes or evolution of spatial objects over time. Each formalism is equipped with different expressiveness, so Boolean semantics and quantitative semantics need to be provided.

Many works have been done on the axiomatization and completeness of modal logics. Patrick Blackburn [4] present the completeness of normal modal logic through maximal consistent set and canonical model. J.M. Davoren [5] proposes topological semantics for intuitionistic tense logics and multimodal logic and provide the Hilbert-style axiomatization and the completeness result. F.D. David [6] prove the absolute completeness of $\mathcal{S} 4_{u}$ for its measure-theoretic semantics. In this paper, we present an axiomatization system for STSL and provide the soundness and completeness result of the axiomatization system.

In this work, there are three contributions:

1) We propose a spatio-temporal specification language STSL, based on STL and $\mathcal{S} 4_{u}$, to specify the changes in topological space and dense time,

2) We interpret the STSL language from two formalisms: $S T S L_{P C}$ and $S T S L_{O C}$, and provide Boolean semantics and quantitative semantics for the language, 
3) We present an axiomatization system and provide the completeness result for the proposed language STSL.

The next section introduces temporal logic STL and modal spatial logic $\mathcal{S} 4_{u}$. Section 3 presents the spatio-temporal specification language STSL and section 4 presents an axiomatization system for the language STSL. In section 5, we conclude the work and talk about the future work.

\section{Signal Temporal LOGIC AND $\mathcal{S} 4_{u}$}

The section provides the background to the proposed spatiotemporal logic, including signal temporal logic (STL) and spatial logic $\mathcal{S} 4_{u}$.

\section{A. Signal Temporal Logic (STL)}

An STL signal [2], [7] is defined on dense-time domain $\mathbb{T}$. A signal function $\varepsilon: \mathbb{T} \rightarrow \mathbb{E}$ associates a set of time domain with a set of signals. Signals with $\mathbb{E}=\mathbb{B}=\{0,1\}$ are called Boolean signals, while those where $\mathbb{E}=\mathbb{R}^{+}$are called realvalued or quantitative signals. A Boolean signal, transformed from real-valued one, can be represented by Metric Temporal Logic [8], [9]. The comparison of some temporal logics is listed in Table I.

TABLE I

THE COMPARISON OF LTL, MTL AND STL

\begin{tabular}{|c|c|c|}
\hline Logic & Time domain & Variable \\
\hline Linear Temporal Logic (LTL) & Discrete time & Boolean value \\
\hline Metric Temporal Logic (MTL) & Dense-time & Boolean value \\
\hline Signal Temporal Logic (STL) & Dense-time & Real-value \\
\hline
\end{tabular}

An execution trace $w$ is a set of real-valued signals $x_{1}^{w}, \ldots, x_{k}^{w}$ bound in some interval $I$ of $\mathbb{R}^{+}$, which is called the time domain of $w$ [3]. Such an interval $I \subseteq \mathbb{R}^{+}$is open $\left(t_{1}, t_{2}\right)$, half-closed and half-open $\left[t_{1}, t_{2}\right)$, half-open and halfclosed $\left(t_{1}, t_{2}\right]$ or closed time interval $\left[t_{1}, t_{2}\right]$. For instance, a running car checks in and enters a highway at $t_{1}$ and checks out and leaves it at $t_{2}$. We say, the car keeps running on the highway in the interval $\left[t_{1}, t_{2}\right]$.

The syntax of STL is given by

$$
\varphi::=\top\left|x_{i} \geq 0\right| \neg \varphi\left|\varphi_{1} \wedge \varphi_{2}\right| \varphi_{1} \mathcal{U}_{I} \varphi_{2}
$$

where $x_{i} \geq 0$ is an atomic predicate whose truth value is determined by the sign of an evaluation based on a signal $x_{i}$. The Boolean operators $\neg$ and $\wedge$ are negation and conjunction, respectively. The time bounded until operator $\mathcal{U}_{I}$ is defined on the time interval $I$.

The formula $\varphi_{1} \vee \varphi_{2}, \diamond_{I} \varphi$ and $\square_{I} \varphi$ can be defined by:

$$
\begin{gathered}
\varphi_{1} \vee \varphi_{2}=\neg\left(\neg \varphi_{1} \wedge \neg \varphi_{2}\right) \\
\diamond_{I} \varphi=\top \mathcal{U}_{I} \varphi, \\
\square_{I} \varphi=\neg \diamond_{I} \neg \varphi .
\end{gathered}
$$

Formula $\diamond_{I} \varphi$ indicates that at some time $t \in I, \varphi$ is eventually satisfiable, while $\square_{I} \varphi$ denotes that $\varphi$ is always satisfiable at each time $t \in I$.

\section{B. An axiomatisation system for STL}

The fundamental composition of the axiomatization system for STL contains all the tautologies like atomic proposition, boolean and quantitative operator in first-oder logic. Temporal expressiveness and inference rules are shown as follows:

A0 All classical tautologies of first-order logic

A1 $\square(\phi \rightarrow \varphi) \rightarrow(\square \phi \rightarrow \square \varphi)$

A2 $\neg \circ \phi \leftrightarrow \circ \neg \phi$

A3 $\circ(\phi \rightarrow \varphi) \rightarrow(\circ \phi \rightarrow \circ \varphi)$

A4 $\square(\phi \rightarrow \circ \varphi) \rightarrow(\phi \rightarrow \square \varphi)$

A5 $(\phi U \varphi) \leftrightarrow \varphi \vee \circ(\phi U \varphi)$

A6 $(\phi U \varphi) \rightarrow \diamond \varphi$

$\operatorname{MP} \frac{\phi \quad \phi \rightarrow \varphi}{\varphi}$

$N_{\square} \frac{\phi}{\vdash \square \phi}$

$N_{\circ} \frac{\phi}{\vdash \circ \phi}$

C. Spatial Logic: $\mathcal{S} 4_{u}$

$\mathcal{S} 4$ [10] is a proposition modal logic and $\tau$ are spatial terms under the topological space interpretation. In the absence of ambiguity, the terminology spatial terms denote spatial objects. According to an observation by [11], $\mathcal{S} 4$ is a logic of topological spaces, and the propositional variable $p$ is interpreted as a subset of the topological space. From the perspective of the topological space, propositional variables of $\mathcal{S} 4$ will be understood as spatial variables [12]. The formula of $\mathcal{S} 4$ is defined as follows:

$$
\tau::=p|\bar{\tau}| \tau_{1} \sqcap \tau_{2} \mid \mathbb{I}_{\tau}
$$

where $p$ is named as spatial variables and $\bar{\tau}$ is the complementary of $\tau, \tau_{1} \sqcap \tau_{2}$ the intersection operation of $\tau_{1}$ and $\tau_{2}$. II is an interior operator under the topological space interpretation. The union and closure operator can be defined by:

$$
\tau_{1} \sqcup \tau_{2}=\overline{\left(\overline{\tau_{1}} \sqcap \overline{\tau_{2}}\right)}, \quad \mathbb{C}_{\tau}=\overline{\mathbb{I}_{\bar{\tau}}}
$$

$\mathbb{C}_{\tau}$ refers to the closure of a spatial object $\tau$. For example, In order to ensure safety, the rear car $c_{\text {rear }}$ can't reach the edge of the front car, the formula can be described by $\overline{\mathbb{C}_{c_{\text {rear }}} \sqcap \mathbb{C}_{c_{\text {front }}}}$.

A topological model is a pair of the form $\mathfrak{M}=(\mathfrak{L}, \mathfrak{V}(p))$, where $\mathfrak{L}=(U, \mathbb{I})$ is a topological space. $U$ is a nonempty set denoting the universe of the space, and $\mathbb{I}$ is the interior operator on $\mathrm{U} \cdot \mathfrak{V}(p)$, as a set of valuations on spatial variables, is a subset of $U$. Therefore we get the valuation of other spatial formulas as follows:

$$
\begin{gathered}
\mathfrak{V}(\bar{\tau})=\mathrm{U}-\mathfrak{V}(\tau), \mathfrak{V}\left(\tau_{1} \sqcup \tau_{2}\right)=\mathfrak{V}\left(\tau_{1}\right) \cup \mathfrak{V}\left(\tau_{2}\right), \\
\mathfrak{V}\left(\mathbb{I}_{\tau}\right)=\mathbb{I V V}(\tau), \mathfrak{V}\left(\tau_{1} \sqcap \tau_{2}\right)=\mathfrak{V}\left(\tau_{1}\right) \cap \mathfrak{V}\left(\tau_{2}\right), \\
\mathfrak{V}\left(\mathbb{C}_{\tau}\right)=\mathbb{C} \mathfrak{V}(\tau) .
\end{gathered}
$$

A spatial logic is a formal language interpreted over a class of structures featuring geometrical entities and relations. Among the well-known spatial logics such as RCC-8 [13], [14], BRCC-8 and $\mathcal{S} 4_{u}$, the most expressive spatial formalism is $\mathcal{S} 4_{u}$ [15]. $\mathcal{S} 4_{u}$ extends $\mathcal{S} 4$ with the universal and existential quantifiers $\forall, \Leftrightarrow$ based on a spatial term $\tau$. $\forall \tau$ refers to that 
there is at least one element in space $\tau ; \forall \tau$ means that all elements in the space belong to $\tau$ ). The formula $\varphi$ is defined in the form of BNF:

$$
\varphi::=\forall \tau|\neg \varphi| \varphi_{1} \wedge \varphi_{2}
$$

where, $\neg \varphi$ is the negation of $\varphi$ and $\varphi_{1} \wedge \varphi_{2}$ the conjunction of $\varphi_{1}$ and $\varphi_{2}$. Correspondingly, the disjunction and existential operator can be defined by:

$$
\varphi_{1} \vee \varphi_{2}=\neg\left(\neg \varphi_{1} \wedge \neg \varphi_{2}\right), \hat{\nabla} \tau=\neg \forall \bar{\tau}
$$

The axiomatization system for $\mathcal{S} 4_{u}$ includes the classical propositional logic in topology $\mathfrak{L}$, the modal logics $\mathrm{K}, \mathrm{T}, 4$ and $\neg$ and inference rules.

$\mathrm{CP}$ axioms of classical propositional logic in $\mathfrak{L}$

$\square K \quad \square(\phi \rightarrow \varphi) \rightarrow(\square \phi \rightarrow \square \varphi)$

$\square T \quad \square \phi \rightarrow \phi$

$\square 4 \square \phi \rightarrow \square \square \phi$

$\neg \neg \diamond \phi \leftrightarrow \square \neg \phi$

and the inference rules

MP $\frac{\phi \phi \rightarrow \varphi}{\varphi}$
$N_{\square} \frac{\phi}{\vdash \square \phi}$
$N_{\diamond} \frac{\phi}{\vdash \diamond \phi}$

\section{Spatio-TEMPoral Specification LANGUAGE}

STL provides an approach that combines the truth value and quantitative value of general signals. But it is inadequate to represent the changes of a spatial entity and the binary relation between spatial entities and temporal aspects. We propose the spatio-temporal specification language that combines STL and $\mathcal{S} 4_{u}$ to describe the evolution in spatial and temporal domain.

\section{A. Spatio-temporal signal}

A spatio-temporal signal is defined with continuous time and topological space [16], [17]. The time domain $\mathbb{T}$ will usually be a real-valued interval $[0, t]$, where $t \in \mathbb{R}_{\geq 0}$. The signal function is extended to spatial-temporal domain with $\varepsilon: \mathbb{T} \times \mathbb{L} \rightarrow \mathbb{E}$, where $\mathbb{L}$ denotes the topological space. The domain of Boolean and quantitative signals extends the domain of STL signals to topological space.

A spatio-temporal trace $w(t, l)$ provides a notation about execution sequence of time $t$ and space $l$. For a spatio-temporal trace, there are two different interpretations:

- A trace represents a sequence of spatial objects and time point and each point in the trace evaluates a pair of spatial objects and time.

- Another interpretation means that a spatio-temporal trace takes spatial objects as the basic entities and spatiotemporal primitive relations could be obtained by the changes of ontology of space over time.

In this work, we treat the spatio-temporal trace as the second interpretation. The changes of spatial objects are influenced by the flow of time.
Definition III.1 (Spatio-temporal signal). A spatio-temporal signal $\varepsilon(t, l)$ is an evaluation of spatial entities in a trace $w$ over time. A Boolean signal $\mu(t, l)$ is an evaluation of an atomic proposition transferred from quantitative signals $\boldsymbol{x}(t, l)$ by atomic predicate $\mu(t, l)=(\boldsymbol{x}(t, l) \geq 0)$ in a trace $w$.

$$
\varepsilon(t, l):=\left\{\begin{array}{c}
\mu(t, l) \quad \text { if } \mathbb{T} \times \mathbb{L} \rightarrow \mathbb{B} \\
\mathbf{x}(t, l) \text { if } \mathbb{T} \times \mathbb{L} \rightarrow \mathbb{R}_{\geq 0}
\end{array}\right.
$$

where boolean function $\mu: \mathbb{T} \times \mathbb{L} \rightarrow \mathbb{B}$ gives rise to the Boolean signals $\varepsilon(t, l)=\mu(t, l)$, while quantitative signals are obtained as the real-valued function $\mathbf{x}: \mathbb{T} \times \mathbb{L} \rightarrow \mathbb{R}_{\geq 0}$, with $\varepsilon(t, l)=\mathbf{x}(t, l)$.

\section{B. The interpretation of the combined logic}

It is essential that a combined spatio-temporal formalism should be provided with enough expressiveness to contain the three parameters [12]:

1) the expressiveness of the spatial component;

2) the expressiveness of the temporal component;

3) the interaction between the two components allowed in the combined logic.

The interaction between the spatial and temporal components should comply with the principle of $P C$ and $O C$, which is used to evaluate the interaction:

1) $S T S L_{P C}$ : the language should be able to express changes over time of the truth-values of purely spatial propositions.

2) $S T S L_{O C}$ : the language should be able to express changes or evolution of spatial objects over time.

$S T S L_{P C}$ expresses the change of truth-value of proposition and it is the elementary requirement for a combined spatiotemporal logic. For instance:

* Eiffel Tower is located in Paris.

* Two cars running on the road never occupy the same location simultaneously.

For $S T S L_{O C}$, spatio-temporal properties are described about the changes of spatial objects over time. We use spatial objects in topological space to interpret the principle such as

* One wave gradually formed in the sea and disappeared eventually on the shore.

* If train A will pass the location in one hour that train B occupies now on the railway, train B must run within one hour.

* A package from a courier service company will eventually be delivered to its destination through several transfer stations.

The spatio-temporal signals are divided into Boolean and quantitative signals. According to the category of spatiotemporal signals, we will present syntax and semantics for the proposed spatio-temporal specification language from two sides:

- The Boolean semantics returns true if the trace of spatiotemporal model satisfies the properties described by $S T S L_{P C}$ formulas. 
- The quantitative semantics returns a real value in different time that can be interpreted as an evaluation of satisfaction of the $S T S L_{O C}$ formulas.

The Boolean semantics of the spatio-temporal specification language interprets that a formula of STSL over spatiotemporal traces returns true or false, so it is able to express changes of the truth-values concerning purely spatial propositions of $P C$. Meanwhile, the $\mathcal{U}_{I}$ and $\square_{I}$ operators of the quantitative semantics are able to express changes or evolution of spatial objects over some fixed finite periods and the whole duration of time of $O C$, respectively. So, the expressiveness power of the combined spatio-temporal logic is enough to describe the spatio-temporal behavior.

\section{The syntax of $S T S L_{P C}$}

We extend the real-value interval into spatio-temporal domain. Formally, we define the spatial temporal interval $I$ as $\left[(t, l),\left(t^{\prime}, l^{\prime}\right)\right], \forall t, t^{\prime} \in \mathbb{T}$ and $t<t^{\prime}$. The $S T S L_{P C}$ is defined on spatio-temporal terms $\tau$ over the spatio-temporal interval $I$, combining temporal logic STL and modal spatial logic $\mathcal{S} 4_{u}$. The syntax of $S T S L_{P C}$ is given by:

$\tau::=p|\bar{\tau}| \tau_{1} \sqcap \tau_{2} \mid \mathbb{I} \tau$

$\varphi::=\forall \tau\left|x_{i} \geq 0\right| \neg \varphi\left|\varphi_{1} \wedge \varphi_{2}\right| \varphi_{1} \mathcal{U}_{I} \varphi_{2}$

- $\tau$ is a spatio-temporal term,

- $p$ is a spatio-temporal variable,

- $\bar{\tau}$ is the complementary of $\tau$,

- $\tau_{1} \sqcap \tau_{2}$ is the intersection of $\tau_{1}$ and $\tau_{2}$,

- II is the interior operator under the topological space interpretation. Moreover, the dual operator of $\mathbb{I}$ is the closure operator $\mathbb{C}$, which means possible or consistent,

- $x_{i} \geq 0$ is an atomic predicate,

- $\neg, \vee$ and $\wedge$ are the Boolean operators,

- $\mathcal{U}_{I}$ is the until operator.

We can define equivalence of operators as syntactic abbreviations:

$$
\begin{gathered}
\mathbb{C} \tau=\mathrm{U}-\mathbb{I} \tau \\
\tau_{1} \sqcup \tau_{2}=\overline{\overline{\tau_{1}} \sqcap \overline{\overline{\tau_{2}}}} \\
\varphi_{1} \vee \varphi_{2}=\neg\left(\neg \varphi_{1} \wedge \neg \varphi_{2}\right) \\
\bigcirc_{I} \varphi=\perp \mathcal{U}_{I} \varphi \\
\diamond_{I} \varphi=\top \mathcal{U}_{I} \varphi \\
\square_{I} \varphi=\neg \diamond_{I} \neg \varphi .
\end{gathered}
$$

Atomic predicates, Boolean operators, and the spatiotemporal bounded until operator $\mathcal{U}_{I}$ are from STL. The new spatial operators are the interior operator $\mathbb{I}$, the closure operator $\mathbb{C}$ with reference to $\mathcal{S} 4_{u}$. The $\bigcirc_{I}, \diamond_{I}$ and $\square_{I}$ operators are derived unary operators. $\square_{I} \varphi$ denotes that $\varphi$ holds within the whole trace of spatio-temporal interval $I$, and $\diamond_{I} \varphi$ means that $\varphi$ holds in at least one time point of the spatio-temporal interval $I$.

\section{The semantics of $S T S L_{P C}$}

A spatio-temporal model is defined on topological space and temporal model. Formally, a spatio-temporal mdoel $\mathfrak{M}=$ $(\mathfrak{T}, \mathfrak{L}, \mathfrak{V})$, where
- $\mathfrak{T}$ is a pair $(\mathbb{T},<)$, where $\mathbb{T}$ is a set of time point and $<$ $\mathrm{R}$ an irreflexive, transitive and asymmetric relation on $\mathbb{T}$ with a linear strict time flow,

- $\mathfrak{L}$ is a topological space domain with the definition of $\mathrm{U}, \mathbb{I}$ in which $\mathrm{U}$ is a nonempty set, the universe of the space, and $\mathbb{I}$ is the interior operator on $U$ satisfying the standard Kuratowski axioms: $\forall X, Y \subseteq \mathrm{U}, \mathbb{I}(X \cap Y)=$ $\mathbb{I} \cap \mathbb{Y}, \mathbb{I} X \subseteq \mathbb{I} \mathbb{I} X$ and $\mathbb{I}(\mathrm{U})=\mathrm{U}$,

- $\mathfrak{V}$ is a valuation on the time point set $\mathfrak{T}$ and the spatial variable set $\mathbb{P}$, i.e., $\forall p \in \mathbb{P}$, and $t \in \mathfrak{T}$ and $\mathfrak{U}(p, t)$ which means the space occupied by $p$ at time point $t$. As for the spatial term $\tau$, the valuation can be defined as: $\mathfrak{V}(\bar{\tau}, t)=$ $\mathrm{U}-\mathfrak{V}(\tau, t), \mathfrak{V}\left(\tau_{1} \sqcap \tau_{2}, t\right)=\mathfrak{V}\left(\tau_{1}, t\right) \cap \mathfrak{V}\left(\tau_{2}, t\right), \mathfrak{V}(\mathbb{I} \tau, t)=$ $\mathbb{I} \mathfrak{V}(\tau, t)$.

We define a spatio-temporal trace $w$ as the changes of spatial objects over time. In spatio-temporal model $\mathfrak{M}, \mathfrak{V}(p)$ evaluates the spatial object $p$. Further, the spatio-temporal trace $w$ represents $\mathfrak{V}(p)$ changes over time $t$, where $t$ belongs to the domain $\mathbb{T}$.

The satisfaction relation for a $S T S L_{P C}$ formula $\varphi$ over a spatio-temporal model $\mathfrak{M}$ is given by:

- $(\mathfrak{M}, t)=\forall \tau \Leftrightarrow \mathfrak{V}(\forall \tau, t)=\top$

- $(\mathfrak{M}, t)=x_{i} \geq 0 \Leftrightarrow \mathfrak{V}\left(x_{i} \geq 0, t\right)=x_{i}$

- $(\mathfrak{M}, t) \models \neg \varphi \Leftrightarrow(\mathfrak{M}, t) \forall \varphi$

- $(\mathfrak{M}, t) \models \varphi_{1} \wedge \varphi_{2} \Leftrightarrow(\mathfrak{M}, t) \models \varphi_{1}$ and $(\mathfrak{M}, t) \models \varphi_{2}$

- $(\mathfrak{M}, t)=\varphi_{1} \mathcal{U}_{I} \varphi_{2} \quad \Leftrightarrow \quad \exists t^{\prime} \in t+I$ s.t. $\left(\mathfrak{M}, t^{\prime}\right) \models$ $\varphi_{2}$ and $\forall t^{\prime \prime} \in\left[t, t^{\prime}\right],\left(\mathfrak{M}, t^{\prime \prime}\right)=\varphi_{1}$

A model $\mathfrak{M}$ satisfies $\varphi$ in $t$, denoted by $(\mathfrak{M}, t) \models \varphi$.

For a given formula $\varphi$ and execution trace $w$, we define the satisfaction signal $\chi(\varphi, w, t, l)$ over a trace $w(t, l)$ :

$$
\forall t \in I, \chi(\varphi, w, t, l):=\left\{\begin{array}{cr}
\top & \text { if }(\mathfrak{M}, t) \models \varphi \\
\perp & \text { otherwise }
\end{array}\right.
$$

In order to compute the satisfaction of a formula $\varphi$, we divide the formula $\varphi$ into each subformula $\phi_{i}$ until atomic formula so that formula $\varphi$ can be computed through the subformula and atomic formulas instead of the entire satisfaction signal $\chi(\varphi, w, t, l)$. The procedure can be treated as a hierarchical structure from the full formula $\varphi$ down to each atomic formula.

E. $S T S L_{O C}$

The difference between $S T S L_{P C}$ and $S T S L_{O C}$ is that $S T S L_{P C}$ involves in the change of truth-values of propositions, while $S T S L_{O C}$ describes the change of extensions of predicates. The syntax of $S T S L_{O C}$ is given by:

$$
\begin{aligned}
& \tau::=p|\bar{\tau}| \tau_{1} \sqcap \tau_{2}|\mathbb{I} \tau| \tau_{1} \mathcal{U}_{I} \tau_{2} \\
& \varphi::=\forall \tau\left|x_{i} \geq 0\right| \neg \varphi\left|\varphi_{1} \wedge \varphi_{2}\right| \varphi_{1} \mathcal{U}_{I} \varphi_{2}
\end{aligned}
$$

The change exists in that the operator $\mathcal{U}_{I}$ can be applied to spatial terms $\tau$. $\tau_{1} \mathcal{U}_{I} \tau_{2}$ refers to $\tau_{1}$ holds until $\tau_{2}$ holds within the interval $I$. Similar to the atomic formulas, the $\bigcirc_{I}$, $\square_{I}$ and $\diamond_{I}$ of spatio-temporal term can also be derived from the operator $\mathcal{U}_{I}$. The unary operators $\square_{I}$ and $\diamond_{I}$ in $S T S L_{O C}$ formulas fuse the $\square_{I}$ and $\diamond_{I}$ operators of STL with that of $\forall$ and $\vartheta$ operators of $\mathcal{S} 4_{u}$. 
A spatio-temporal trace $w$ is a sequence over signals $\varepsilon$. The Boolean satisfaction relation and satisfaction degree for a $S T S L_{O C}$ formula $\varphi$ over a spatio-temporal trace $w$ is similar to that of $S T S L_{P C}$ formula.

We define $\rho$ to quantify the satisfaction degree of the property $\varphi$ over the trace $w(t, l)$, and it returns a real number $\rho(\varphi, w, t, l)$. The quantitative satisfaction relation for a formula $\varphi$ over a spatio-temporal trace $w$ is given by:

- $\rho(\forall \tau, w, t, l)=\top$

- $\rho\left(x_{i} \geq 0, w, t, l\right)=f(w(t, l))$ where $\mu \equiv(f \geq 0)$

- $\rho(\neg \varphi, w, t, l)=-\rho(\varphi, w, t, l)$

- $\rho\left(\varphi_{1} \wedge \varphi_{2}, w, t, l\right)=\min \left\{\rho\left(\varphi_{1}, w, t, l\right), \rho\left(\varphi_{2}, w, t, l\right)\right\}$

- $\rho\left(\varphi_{1} \vee \varphi_{2}, w, t, l\right)=\max \left\{\rho\left(\varphi_{1}, w, t, l\right), \rho\left(\varphi_{2}, w, t, l\right)\right\}$

- $\rho\left(\square_{I} \varphi, w, t, l\right)=\inf _{t^{\prime} \in t+I}\left\{\rho\left(\varphi, w, t^{\prime}, l^{\prime}\right)\right\}$

- $\rho\left(\diamond_{I} \varphi, w, t, l\right)=\sup _{t^{\prime} \in t+I}\left\{\rho\left(\varphi, w, t^{\prime}, l^{\prime}\right)\right\}$

- $\rho\left(\varphi_{1} \mathcal{U}_{I} \varphi_{2}, w, t, l\right)=\sup _{t^{\prime} \in t+I}\left(\min \left\{\rho\left(\varphi_{2}, w, t^{\prime}, l^{\prime}\right)\right.\right.$, $\left.\inf _{t^{\prime \prime} \in\left[t, t^{\prime}\right]}\left(\rho\left(\varphi_{1}, w, t^{\prime \prime}, l^{\prime \prime}\right)\right\}\right)$

The intuitive meaning for $\rho\left(\square_{I} \varphi, w, t, l\right)$ refers to that we achieve the infimum of $\rho\left(\varphi, w, t^{\prime}, l^{\prime}\right), \forall t^{\prime} \in t+I$ over the trace. Similar to $\rho\left(\square_{I} \varphi, w, t, l\right), \rho\left(\diamond_{I} \varphi, w, t, l\right)$ returns the supremum of $\rho\left(\varphi, w, t^{\prime}, l^{\prime}\right), \forall t^{\prime} \in t+I$.

Especially, if $x_{i} \geq 0$, the satisfaction signal will be

$$
\forall t \in I, \chi\left(x_{i} \geq 0, w, t, l\right):= \begin{cases}\top & \text { if } x_{i} \geq 0 \\ \perp & \text { otherwise }\end{cases}
$$

The connection is built between Boolean signals and quantitative signals by the way of predicate $x_{i} \geq 0$ and obtain the satisfaction signal $\chi\left(x_{i} \geq 0, w, t, l\right)$. In the quantitative semantics, however, atomic predicates $x_{i} \geq 0$ do not evaluate to $T$ or $\perp$ but give a real value of the quantitative signals $x_{i}$ by satisfaction degree $\rho(\varphi, w, t, l)$ representing the distance to satisfaction or not.

\section{AN AXIOMATIZATION SYSTEM FOR STSL}

STSL is a logical system. A proof in STSL is a sequence of finite formula: $A_{0}, A_{1}, \ldots, A_{n}$, where each of them is a axiom, or there exists $j, k<i$, such that $A_{i}$ is the conclusion derived from $A_{j}$ and $A_{k}$ using $M P$ inference rule. The last term $A_{n}$ is a theorem in STSL, using the sign $\vdash A_{n}$, where $n$ is the length of proof.

The notions of deducibility and consistency [4], [18] is fundamental to deduce the logic system STSL. A formula $A$ is deducible from a set of formulas $\Gamma$ in a system $\mathcal{S T}$, written $\Gamma \Vdash_{\mathcal{S T}} A$, if and only if $\mathcal{S} \mathcal{T}$ contains a theorem of the form $\left(A_{1} \wedge \ldots \wedge A_{n}\right) \rightarrow A$, where the conjuncts $A_{i}(i=1, \ldots, n)$ of the antecedent are formulas in $\Gamma$. A set of formulas $\Gamma$ is consistent in $\mathcal{S T}$, written $\operatorname{Con}_{\mathcal{S} \mathcal{T}} \Gamma$, just in case the formula $\perp$ is not $\mathcal{S} \mathcal{T}$-deducible from $\Gamma$.

Definition IV.1 (ST-MCS). A set of formulas $\Gamma$ is maximal $\mathcal{S T}$-consistent iff

(i) $\Gamma$ is $\mathcal{S} \mathcal{T}$-consistent, and

(ii) for every formula $A$, if $\Gamma \cup\{A\}$ is $\mathcal{S} \mathcal{T}$-consistent, then $A \in \Gamma$.
If $\Gamma$ is a maximal $\mathcal{S T}$-consistent set of formulas then we say it is an $\mathcal{S T}$-MCS. The (ii) condition refers to that any set of formulas properly containing $\Gamma$ is $\mathcal{S} \mathcal{T}$-inconsistent.

The canonical model defined by [18] to induce the soundness and completeness of modal logics. We extend the notation of canonical model to spatio-temporal systems for completeness of STSL.

Definition IV.2 (ST -canonical Model). The $\mathcal{S} \mathcal{T}$-canonical model $\mathfrak{M}^{\Gamma}$ for a spatio-temporal logic is a triple $\left(W^{\Gamma}, R^{\Gamma}, V^{\Gamma}\right)$ where:

(i) $W^{\Gamma}$ is the set of all $\Gamma-M C S s$;

(ii) $R^{\Gamma}$ is a topological relation on topological space over a quasi-order on time. It is the canonical binary relation on $W^{\Gamma}$ defined by $s R_{i}^{\Gamma} s^{\prime}$ over state $s$ and $s^{\prime}$ if for all formulas $\phi, \phi \in s$ implies $\phi \in s^{\prime}$.

(iii) $V^{\Gamma}$ is the valuation defined by $V^{\Gamma}(p)=\left\{s \in W^{\Gamma} \mid p \in\right.$ $s\} . V^{\Gamma}$ is called the canonical valuation.

Lemma IV.1 (Truth Lemma). Let $\mathcal{S T}$-canonical model be a class of tt-model. For all $\phi \in \mathcal{S T}$-MCS, $\mathcal{S T} \Vdash \phi$ iff $\phi \in \mathcal{S T}$ MCS.

Proof. The proof is by induction on the structure of $\phi$.

Base case: Suppose $\phi$ is a spatial formula $\forall \tau$ or an atomic predicate $x_{i} \geq 0$.

$(\mathcal{S T}, s) \Vdash \forall \tau \Leftrightarrow V^{\Gamma}(\forall \tau, s)=\top \Leftrightarrow \forall \tau \in s$,

$(\mathcal{S} \mathcal{T}, s) \Vdash x_{i} \geq 0 \Leftrightarrow V^{\Gamma}\left(x_{i} \geq 0, s\right)=x_{i} \Leftrightarrow x_{i} \geq 0 \in s$.

Inductive step: Suppose $\phi$ is an atomic predicate $\neg \phi, \phi_{1} \wedge$ $\phi_{2}, \phi_{1} \vee \phi_{2}, \square \phi, \diamond \phi, \phi \mathcal{U} \varphi$. We show the proof of the case $\square \phi$, and leave the others to reader. We have $(\mathcal{S T}, s) \Vdash \square \phi \Leftrightarrow$ $\square \phi \in s$ (assuming the inductive hypothesis).

$(\mathcal{S T}, s) \Vdash \square \phi$

$\Leftrightarrow \forall s^{\prime}, s R^{\Gamma} s^{\prime} \Rightarrow \mathcal{S T}, s^{\prime} \Vdash \phi$

$\Leftrightarrow \forall, s R^{\Gamma} s^{\prime} \Rightarrow \phi \in s^{\prime}$

we need to show that $\square \phi \in s \Leftrightarrow \forall s^{\prime}, s R^{\Gamma} s^{\prime} \Rightarrow \phi \in s^{\prime}$. $\Rightarrow$ follows immediately from the definition IV.2.

As for $\Leftarrow$ : suppose $\square \phi \notin s$. We need to show

$\exists s^{\prime}, s R^{\Gamma} s^{\prime}$ and $\phi \notin s^{\prime}$

$\Leftrightarrow \exists s^{\prime}, s R^{\Gamma} s^{\prime}$ and $\neg \phi \in s^{\prime}$

$\Leftrightarrow \exists s^{\prime},\{\varphi \mid \square \varphi \in s\} \subseteq s^{\prime}$ and $\neg \phi \in s^{\prime}$

$\Leftrightarrow \exists s^{\prime},\{\varphi \mid \square \varphi \in s\} \cup\{\neg \phi\} \subseteq s^{\prime}$

It is easy to show that $\{\varphi \mid \square \varphi \in s\} \cup\{\neg \phi\}$ is $\mathcal{S T}$ consistent. Suppose not, i.e., $\{\varphi \mid \square \varphi \in s\} \cup\{\neg \phi\}$ is $\mathcal{S T}$-inconsistent. Then $\vdash_{\mathcal{S T}}\left(\varphi_{1} \wedge \ldots \wedge \varphi_{n}\right) \rightarrow \phi$ for some $\left\{\square \varphi_{1}, \ldots \square \varphi_{n}\right\} \subseteq s$. But $\mathcal{S T}$ is canonical and $s$ is $\mathcal{S} \mathcal{T}$-MCS, so $s$ must contain $\left(\square \varphi_{1} \wedge \ldots \wedge \varphi_{n}\right) \rightarrow \square \phi$. From $\square \varphi_{i} \in s$, it follows $\square \phi \in s$. This contradicts the hypothesis that $\square \phi \notin s$

Definition IV.3. The Hilbert-style proof system for the logic STSL has the following axiom schemes:

A0 All classical tautologies of first-order logic

A1 $\neg \circ \phi \leftrightarrow \circ \neg \phi$

A2 $\neg \diamond \phi \leftrightarrow \square \neg \phi$ 


$$
\begin{aligned}
& \text { A3 }(\phi \rightarrow \varphi) \rightarrow(\circ \phi \rightarrow \circ \varphi) \\
& \text { A4 } \square(\phi \rightarrow \circ \varphi) \rightarrow(\phi \rightarrow \square \varphi) \\
& \text { A5 }(\phi U \varphi) \leftrightarrow \varphi \vee \circ(\phi U \varphi) \\
& \text { A6 }(\phi U \varphi) \rightarrow \diamond \varphi \\
& \square K \quad \square(\phi \rightarrow \varphi) \rightarrow(\square \phi \rightarrow \square \varphi) \\
& \square T \quad \square \phi \rightarrow \phi \\
& \square 4 \quad \square \phi \rightarrow \square \square \phi
\end{aligned}
$$

And the inference rules:

$$
\begin{aligned}
& \text { MP } \frac{\phi \phi \rightarrow \varphi}{\varphi} \\
& N_{\square} \frac{\phi}{\vdash \square \phi} \\
& N_{\diamond} \frac{\phi}{\vdash \diamond \phi}
\end{aligned}
$$

Soundness refers to that all the theorems in STSL are logically valid. Equivalently, A spatio-temporal logic is sound with respect to $t t$-model if for all the formula $\phi, \vdash_{\mathcal{S T} \phi}$ implies $\Vdash \phi$. Let $\mathcal{S T}$ be a class of $t t$-model, A spatio-temporal logic is strongly complete in $\mathcal{S T}$ if for any set of formulas $\Gamma \cup\{\phi\}$, if $\Gamma \Vdash_{\mathcal{S}} \mathcal{T} \phi$ then $\Gamma \vdash_{\mathcal{S T}} \phi$. If the semantics of $\Gamma$ satisfies $\phi$ on $\mathcal{S T}$ then $\phi$ is deducible from $\Gamma$.

Theorem IV.2. The above axiomatization is sound for ttmodel.

Proof. This follows from the fact that all axioms are valid and all rules preserve validity.

Theorem IV.3. The system for STSL is weakly complete with respect to $t$-model. i.e., for every $\boldsymbol{S T S L}$ formula, $\|_{\mathcal{S T}} \phi$ implies $\vdash_{\Gamma} \phi$.

Proof. We will present the proof with weak completeness of STSL based on the work [19], [20]. The strong completeness is equal to frame completeness and compactness in universal modal logic [19]. Temporal logic in the flow of real time has weak completeness [20], which proposes finitely complete and expressively complete, but fails compactness theorem. Further, a complete result based on the lexicographic products of modal logics with linear temporal logic is present in [21]. Those conclusions contribute greatly to the proof of weak completeness.

\section{CONClusion AND FUture WORK}

In this paper, we build a spatio-temporal specification language, combining STL with spatial logic $\mathcal{S} 4_{u}$, specifically containing dense time and topological space. We provide the syntax and semantics of the spatio-temporal language, and guarantee the seamless integration of spatial logic with temporal aspect from the perspective of the changes of purely spatial proposition $S T S L_{P C}$ and spatial objects $S T S L_{O C}$ over time. A Hilbert-style proof axiomatization system and the soundness and completeness result is present for the language.

The proposed STSL has a powerful expressiveness. It will be interesting to find a strongly complete fragment of STSL. For that, a more constrained axiomatization system need to be present, and more restricted inference and proof should be provided.

\section{ACKNOWLEDGMENT}

Our deepest gratitude goes to the anonymous reviewers for their valuable suggestions to improve this paper. This paper is partially supported by funding under National Key Research and Development Project 2017YFB1001800, NSFC 61572195 and Shanghai SHEITC Project 2017-GYHLW-01036.

\section{REFERENCES}

[1] Ehsan Ahmad, Yunwei Dong, Shuling Wang, Naijun Zhan, and Liang Zou. Adding formal meanings to aadl with hybrid annex. In International Conference on Formal Aspects of Component Software, pages 228-247. Springer, 2014.

[2] Vasumathi Raman, Alexandre Donzé, Dorsa Sadigh, Richard M Murray, and Sanjit A Seshia. Reactive synthesis from signal temporal logic specifications. In Proceedings of the 18th international conference on hybrid systems: Computation and control, pages 239-248. ACM, 2015.

[3] Alexandre Donzé, Thomas Ferrere, and Oded Maler. Efficient robust monitoring for stl. In International Conference on Computer Aided Verification, pages 264-279. Springer, 2013.

[4] Patrick Blackburn, Maarten De Rijke, and Yde Venema. Modal Logic: Graph. Darst, volume 53. Cambridge University Press, 2002.

[5] Jennifer M Davoren. Topological semantics and bisimulations for intuitionistic modal logics and their classical companion logics. In International Symposium on Logical Foundations of Computer Science, pages 162-179. Springer, 2007.

[6] David Fernández-Duque. Absolute completeness of $s 4 u$ for its measuretheoretic semantics. Advances in modal logic, 8:100-119, 2010.

[7] Alexandre Donzé and Oded Maler. Robust satisfaction of temporal logic over real-valued signals. In International Conference on Formal Modeling and Analysis of Timed Systems, pages 92-106. Springer, 2010.

[8] Patricia Bouyer. Model-checking timed temporal logics. Electronic Notes in Theoretical Computer Science, 231:323-341, 2009.

[9] Haiying Sun, Jing Liu, Xiaohong Chen, and Dehui Du. Specifying cyber physical system safety properties with metric temporal spatial logic. In Software Engineering Conference (APSEC), 2015 Asia-Pacific, pages 254-260. IEEE, 2015.

[10] Richard E Ladner. The computational complexity of provability in systems of modal propositional logic. SIAM journal on computing, 6(3):467-480, 1977.

[11] John Charles Chenoweth McKinsey. A solution of the decision problem for the lewis systems s2 and s4, with an application to topology. The Journal of Symbolic Logic, 6(4):117-124, 1941.

[12] David Gabelaia, Roman Kontchakov, Agi Kurucz, Frank Wolter, and Michael Zakharyaschev. Combining spatial and temporal logics: expressiveness vs. complexity. Journal of Artificial Intelligence Research, 23:167-243, 2005.

[13] David A Randell, Zhan Cui, and Anthony G Cohn. A spatial logic based on regions and connection. KR, 92:165-176, 1992.

[14] Weiming Liu, Li Sanjiang, and Renz Jochen. Combining rcc-8 with qualitative direction calculi: Algorithms and complexity. In Twenty-First International Joint Conference on Artificial Intelligence, 2009.

[15] Roman Kontchakov, Agi Kurucz, Frank Wolter, and Michael Zakharyaschev. Spatial logic+ temporal logic=? In Handbook of spatial logics, pages 497-564. Springer, 2007.

[16] Laura Nenzi, Luca Bortolussi, Vincenzo Ciancia, Michele Loreti, and Mieke Massink. Qualitative and quantitative monitoring of spatiotemporal properties. In Runtime Verification, pages 21-37. Springer, 2015.

[17] Oded Maler and Dejan Nickovic. Monitoring temporal properties of continuous signals. In Formal Techniques, Modelling and Analysis of Timed and Fault-Tolerant Systems, pages 152-166. Springer, 2004.

[18] Brian F Chellas. Modal logic: an introduction. Cambridge university press, 1980.

[19] Valentin Goranko and Solomon Passy. Using the universal modality: gains and questions. Journal of Logic and Computation, 2(1):5-30, 1992.

[20] Dov M. Gabbay and Ian M. Hodkinson. An axiomatization of the temporal logic with until and since over the real numbers. Journal of Logic and Computation, 1(2):229-259, 1990.

[21] Philippe Balbiani and David Fernández-Duque. Axiomatizing the lexicographic products of modal logics with linear temporal logic. 2016 